\title{
Comparison of Voice Quality and Cost Effectiveness of Endoscopic Cordectomy Using Microdissection Electrodes with Laser Cordectomy and Radiotherapy
}

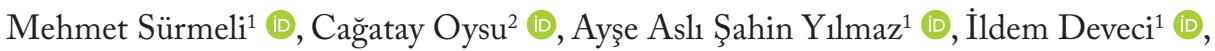 \\ Burak Karabulut $^{1}$ (D), Ahmet Volkan Sunter ${ }^{3}$ (D), Özgür Yiğit ${ }^{3}$ (D), Beyhan Ceylaner Bıcakçı ${ }^{4}$ (]) \\ ${ }^{1}$ Department of Otorhinolaryngology, Health Sciences University, Ümraniye Training and Research Hospital, İstanbul, Turkey \\ ${ }^{2}$ Department of Otorhinolaryngology, Marmara University School of Medicine, İstanbul, Turkey \\ Original Investigation $>{ }^{3}$ Department of Otorhinolaryngology, Health Sciences University, İstanbul Training and Research Hospital, İstanbul, Turkey \\ ${ }^{4}$ Department of Radiation Oncology, Kartal Lütfi Kırdar Training and Research Hospital, İstanbul, Turkey
}

Abstract

\begin{abstract}
ORCID ID of the authors: M.S. 0000-0002-7936-2957; C..0. 0000-0002-6756-8456; A.A.S.Y. 0000-0002-7846-9453; I.D. $0000-0002-1573-8345$; B.K. 0000-0002-3958-3683; A.V.S. 0000-0001-8601-0450; O.Y. 0000-0003-1731-3233; B.C.B. 0000-0003-2509-6742.
\end{abstract}

Cite this article as: Sürmeli M, Oysu Ç, Şahin YIImaz AA, Devecil, Karabulut B, Sunter AV, et al. Comparison of Voice Quality and Cost Effectiveness of Endoscopic Cordectomy Using Microdissection Electrodes with Laser Cordectomy and Radiotherapy. Turk Arch Otorhinolaryngol 2019; 57(1): 1-6.
Objective: To compare the acoustic parameters and the cost effectiveness achieved after endoscopic cordectomy using radiofrequency microdissection electrodes (ECRM) with those of transoral laser cordectomy and radiotherapy.

Methods: The study included 81 disease-free patients previously treated for early larynx carcinoma (30 with ECRM, 27 with transoral laser cordectomy, 24 with primary radiotherapy). Post-treatment voice analysis was performed in all patients. Additionally, the cost effectiveness of all treatment procedures was calculated. Results: The GRBAS (G: Grade, R: Roughness, B: Breathiness, A: Asthenia, and S: Strain) scale showed a significant difference between the groups $(p<0.001)$. The mean values for perceptive assessment score in the radiotherapy group were significantly lower than those in the ECRM group $(p<0.05)$. There were no significant differences between the ECRM group and the transoral laser cordectomy group in terms of the perceptive assessment scores ( $p>0.05$ for all). Percent jitter, percent shim- mer and fundamental frequency (F0) were significantly different in the radiotherapy group than the ECRM and the transoral laser cordectomy groups $(\mathrm{p}<0.05)$. Maximum phonation time (MPT) in the radiotherapy group was significantly longer than the ECRM and transoral laser cordectomy groups $(p<0.001)$. There were no significant differences between the ECRM group and the transoral laser cordectomy group in terms of the MPT ( $p>0.05$ ). The mean cost of the ECRM technique was statistically lower than the other treatment techniques $(p<0.05)$. Moreover, the length of hospital stay after ECRM was statistically significantly shorter than was after laser cordectomy $(\mathrm{p}<0.05)$.

Conclusion: Objective and perceptive voice analysis after ECRM showed comparable results with transoral laser cordectomy, but poorer results than radiotherapy. Cost effectiveness of ECRM was found to be better than the other two treatment techniques

Keywords: Early laryngeal cancer, voice quality, radiofrequency ablation, cost effectiveness

\section{Introduction}

Laryngeal cancer is the most common head and neck cancer with $75 \%$ of cases confined to the glottic level (1). While there are variations in the definition of early glottic cancers given in the literature, the most commonly used definition is the early T stage, such as T1 and T2 (1). Laryngofissure cordectomy, transoral laser surgery and external beam radiotherapy are the treatment modalities of early glottic laryngeal cancers.

Many studies have evaluated the best approach to management of early glottic cancers. Debate continues on the advantages and disadvantages of each modality in terms of local control, larynge- al preservation, survival, functional outcome, and medical costs (2).

Since radiotherapy and transoral laser surgery have become the main treatment methods for early glottic carcinoma, many reports have been published on the optimal therapy for this disease. For superficial midcord T1a lesions, evidence suggest that both modalities have comparable control rates and voice outcomes $(3,4)$. The choice should be based on the preferences of the patient and the clinician, the patient's overall medical condition, and the current health economic environment, the cost of the treatment $(1,5)$. 
Endoscopic transoral laser surgery with $\mathrm{CO}_{2}$ laser is widely used. As an alternative to $\mathrm{CO}_{2}$ laser, endoscopic cordectomy using radiofrequency microdissection electrodes (ECRM) has been defined by Basterra et al. (5) in 2006. The basic principle of this surgical procedure is the application of electrical current with a hard, small and focused metal (with the tip of the micro-electrode) (6). In their various publications Basterra et al. (5-7) have shown this surgery to be a useful, inexpensive alternative to $\mathrm{CO}_{2}$ lasers, with much simpler equipment for the treatment of glottic and selected supraglottic tumors. They also demonstrated that vocal cord tissue damage was similar in both laser cordectomy and microdissection electrode cordectomy (6).

In our study, we aimed to compare the functional outcomes and the cost effectiveness of ECRM, transoral laser cordectomy, and radiotherapy, which are three different treatment modalities in early glottic cancer cases. To our knowledge, no study in English literature has previously compared the outcomes of this method with transoral laser cordectomy and radiotherapy in the treatment of early glottic cancers.

\section{Methods}

\section{Patients}

In the ECRM group, records of patients with T1a glottic cancer treated at the University of Health Sciences, Ümraniye Training and Research Hospital Otolaryngology Clinic between January 2013 and January 2017 were reviewed. Of these patients, those with recurrent or residual disease were excluded. Forty-four patients were eligible for inclusion in the study. Patients were contacted and asked to participate in this study to which 30 agreed.

Between December 2013 and November 2017, patients with single midcord lesions that did not extend to the anterior commissure (early glottic cancer [T1a]) were primarily offered transoral laser cordectomy at the University of Health Sciences, İstanbul Training and Research Hospital Otolaryngology Clinic. Forty-nine patients patients were eligible for inclusion in the study. Patients were contacted and asked to participate in this study to which 27 agreed.

In the radiotherapy group, records of patients with T1a glottic cancer treated at the University of Health Sciences, Kartal Lütfi Kirdar Training and Research Radiation Oncology Clinic between January 2012 and January 2016 were reviewed. Patients in whom the extent of the tumor could not be retrospectively determined and those with recurrent or residual disease were excluded. Forty patients were eligible for inclusion in the study. Patients were contacted and asked to participate in this study to which 24 agreed.

All patients had biopsy results for squamous cell carcinoma. The study protocol was reviewed and approved by the Ethics Committee (reference number 7389, approval date 11.05.15). Informed consent form was obtained from patients who participated in this study.

\section{Treatment Procedures}

Endoscopic Cordectomy Using Radiofrequency Microelectrodes (ECRM): The surgical procedure was performed with direct suspension laryngoscopy. Tissue resection was performed using a 21-cm-long radiofrequency Arrowtip ${ }^{\mathrm{TM}}$ tungsten monopolar needle with a straight and/or $90^{\circ}$ and $180^{\circ}$ angle tip and "CURIS" Radiofrequency Generator (Sutter Medizintechnik, Freiburg, Germany). The generator was adjusted to a power of $15-25$ watts and to the "CUT1" monopolar cutting mode, depending on the response of the electrode to the tissue. Resection was performed as described by Basterra et al. $(5,7)$ and can be summarized as follows: The false vocal cord on the side of the tumor was clamped with forceps and resected with a $180^{\circ}$-angle microelectrode. Resection of the vocal cord was performed using either the same or the $90^{\circ}$ microelectrode by an anteroposterior deep section over the lateral limit of the vocal cord, followed by up-down sections, first one in the anterior commissure, second one in its attachment into the vocal process of the arytenoid cartilage. Endoscopic cordectomy using radiofrequency microelectrodes surgery corresponded to type 3 cordectomy (transmuscular cordectomy) according to European Laryngeal Society (ELS) classification (8).

Transoral Laser Cordectomy: Patients who underwent transoral laser cordectomy were hospitalized one day before the operation in order to complete the official purchase order procedures for the laser equipment consumables. Endoscopic cordectomies were performed under general endotracheal anesthesia with Sharplan Lumenis $40 \mathrm{C} \mathrm{CO}_{2}$ laser (Sharplan Lasers Inc., London, UK). The Carl Zeiss Surgical OPMI Sensera optical microscope (Carl Zeiss Meditec Inc., Dublin, CA, USA) was used to perform this procedure.

External Beam Radiotherapy: The therapy was carried out using a $6 \mathrm{MV}$ photons linear accelerator to bilateral ports and one in front, with field sizes ranging from $5 \times 5$ to $6 \times 6 \mathrm{~cm}$. The radiation dose for treatment of T1 glottic carcinoma was 65-70 Gy (2.0 Gy per fraction). The average duration of radiotherapy was $33 \pm 2$ days.

\section{Assessment of Voice Quality and Function}

Recordings of speech and voice analyzes were made in a soundproof room. During recording, the distance between the microphone (Dynamic Rode ${ }^{\circledR}$ NT1; Rode, Sydney, Australia) and the mouth was at least $20 \mathrm{~cm}$. The speech recording for perceptual analysis consisted of a phonetically balanced Turkish text ( $\mathrm{Ja}^{-}$ le'nin Dünyası [Jale's World]) which was read by each subject in normal tone and recorded. Approximately 3 seconds / a / phonem was recorded for acoustic analysis. In addition, the longest / a / phoneme recorded after deep inspiration was taken to calculate the Aerodynamic voice analysis (maximum phonation time).

Perceptual analysis GRBAS (G: Grade, R: Roughness, B: Breathiness, A: Asthenia, and S: Strain) scale

Perceptual sound analysis was performed with the GRBAS scale. For each parameter, evaluation was made between 0 and 3 points. On this scale, 0 represents normal sound quality, while high scores indicate worse sound quality. Recorded sound samples were evaluated blind by three independent authors. 
Table 1. Patient characteristics

\begin{tabular}{|c|c|c|c|c|c|}
\hline \multirow[b]{2}{*}{ Number of subjects } & & ECRM & Laser Cordectomy & Radiotherapy & \multirow[b]{2}{*}{$\mathrm{p}$} \\
\hline & & 30 & 27 & 24 & \\
\hline $\operatorname{Sex}(\mathrm{M} / \mathrm{F}) ; \mathrm{n}$ & & $28 / 2$ & $27 / 0$ & $22 / 2$ & 0.453 \\
\hline \multirow[t]{2}{*}{ Age (years) } & Mean \pm SD & $65.46 \pm 9.52$ & $60.22 \pm 7.62$ & $62.42 \pm 8.92$ & $0.055_{b}$ \\
\hline & Median (Range) & $65(48-79)$ & $60(47-78)$ & $60(42-74)$ & \\
\hline \multirow[t]{2}{*}{ Follow-up (months) } & Mean \pm SD & $29.16 \pm 6.28$ & $25.18 \pm 7.52$ & $27.87 \pm 3.97$ & $0.17_{b}$ \\
\hline & Median (Range) & $30(18-40)$ & $26(12-37)$ & $28(21-34)$ & \\
\hline Recurrence & & none & none & none & - \\
\hline
\end{tabular}

ECRM: Endoscopic Cordectomy Using Radiofrequency Microelectrodes

${ }^{a}$ Fisher-Freeman-Halton test

${ }^{\mathrm{b}} \mathrm{Kruskal}-$ Wallis test

* $\mathrm{p}<0.05$

Table 2. Comparison of the groups according to the auditory perceptual assessments using the GRBAS (G: Grade, R: Roughness, B: Breathiness, A: Asthenia, and S: Strain) scale

\begin{tabular}{l|c|c|c|c}
\hline & $\begin{array}{c}\text { ECRM } \\
\text { n: } 30\end{array}$ & $\begin{array}{c}\text { Laser } \\
\text { Cordectomy } \\
\text { n: } 27\end{array}$ & $\begin{array}{c}\text { Radiotherapy } \\
\text { n: } 24\end{array}$ & \multirow{2}{*}{} \\
\cline { 2 - 4 } & $\begin{array}{c}\text { Median } \\
\text { (Min-Max) }\end{array}$ & $\begin{array}{c}\text { Median } \\
\text { (Min-Max) }\end{array}$ & $\begin{array}{c}\text { Median } \\
\text { (Min-Max) }\end{array}$ & p \\
\hline $\mathrm{G}$ & $2(1-3)$ & $2(1-2)$ & $1(0-2)$ & ${ }^{\mathrm{a}}<0.001^{*}$ \\
\hline $\mathrm{R}$ & $2(2-3)$ & $2(2-3)$ & $1(0-3)$ & ${ }^{\mathrm{a}}<0.001^{*}$ \\
\hline $\mathrm{B}$ & $2(2-3)$ & $2(1-2)$ & $1(0-1)$ & ${ }^{\mathrm{a}}<0.001^{*}$ \\
\hline $\mathrm{A}$ & $2(1-3)$ & $2(1-3)$ & $1(0-2)$ & ${ }^{a}<0.001^{*}$ \\
\hline $\mathrm{S}$ & $2(2-3)$ & $3(2-3)$ & $1(0-2)$ & ${ }^{\mathrm{a}}<0.001^{*}$ \\
\hline
\end{tabular}

SD: Standard deviation; ECRM: Endoscopic Cordectomy Using Radiofrequency Microelectrodes

${ }^{a}$ Fisher's Exact Test

*p $<0.05$

\section{Acoustic analysis}

The mean fundamental frequencies $\left(\mathrm{F}_{0}\right.$ in Hertz $\left.[\mathrm{Hz}]\right)$, percentage of jitter and percentage of shimmer were determined. Multi-dimensional voice and speech analysis was performed with Praat speech processing software (University of Amsterdam, The Netherlands).

\section{Cost-effectiveness analysis}

For cost-effectiveness analysis, biopsy- and treatment-costs were calculated for every patient. Costs were obtained from the hospital database after the biopsies and the surgical procedures of the ECRM and transoral laser cordectomy groups. Total hospitalization times for each of the two treatment approaches were calculated. Additionally, treatment costs of patients undergoing primary radiotherapy were obtained from the hospital database after their biopsy and outpatient treatment. All treatment costs were calculated with the US Dollar/Turkish Lira exchange rate applicable at the time of treatment. Treatment costs of all groups were compared with statistical analysis methods.

\section{Statistical Analysis}

The differences between the groups for the statistical analysis of this study were calculated with the Statistical Package for So- cial Science for windows version 22.0 (IBM Corp.; Armonk, NY, USA). Descriptive statistical analyses were performed. Data were evaluated for normal distribution with the Kolmogorov-Smirnov Test. Fischer's Exact test was used for statistical analysis of categorical variables. The Kruskal-Wallis Test and the Mann-Whitney U Test were used for the statistical analysis of the quantitative data that were not normally distributed. Statistical significance was set at $\mathrm{p}<0.05$.

\section{Results}

Characteristics of the patients included in the three groups are listed in Table 1.The groups were similar with respect to sex, age and recurrence status.

\section{Perceptual analysis (GRBAS score)}

The mean scores for the perceptive assessment of the voice quality on the basis of the GRBAS scale showed a significant difference between the groups, as presented in Table $2(p<0.001)$. The mean values for all parameters were significantly lower in the radiotherapy group than the ECRM and the transoral laser cordectomy groups $(\mathrm{p}<0.01)$; however, there were no significant differences between the ECRM group and the transoral laser cordectomy group in terms of any of the parameters ( $p>0.05$ for all).

\section{Acoustic analysis}

The findings of the acoustic analysis (comparison of the mean fundamental frequencies (F0 in Hertz [Hz]), percentage of jitter, and percentage of shimmer) in all groups are given in Table 3.

When the acoustic parameters of the three groups were compared, statistically significant differences were found in all parameters between the radiotherapy group and the other treatment procedure groups $(\mathrm{p}<0.05)$.

\section{Aerodynamic efficiency analysis}

Maximum phonation times (MPT) found in all groups are shown in Table 3. In the radiotherapy group MPT was significantly higher than in the ECRM and the transoral laser cordectomy groups $(\mathrm{p}<0.001)$. There was no significant difference between the ECRM group and the transoral laser cordectomy group in terms of the MPT $(\mathrm{p}>0.05)$. 
Table 3. Comparison of the groups according to the acoustic and aerodynamic efficiency analysis

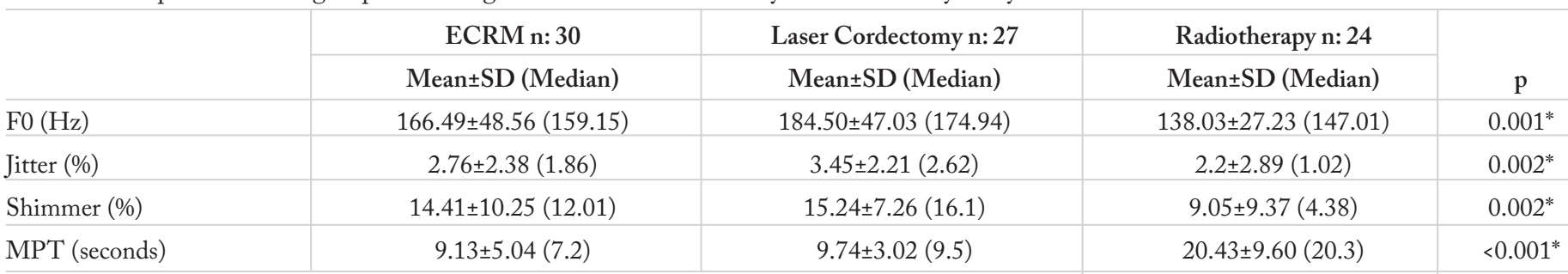

F0: fundamental frequency; MPT: maximum phonation time; ECRM: endoscopic cordectomy using radiofrequency microelectrodes; SD: standard deviation Kruskall-Wallis Test

*p $<0.05$

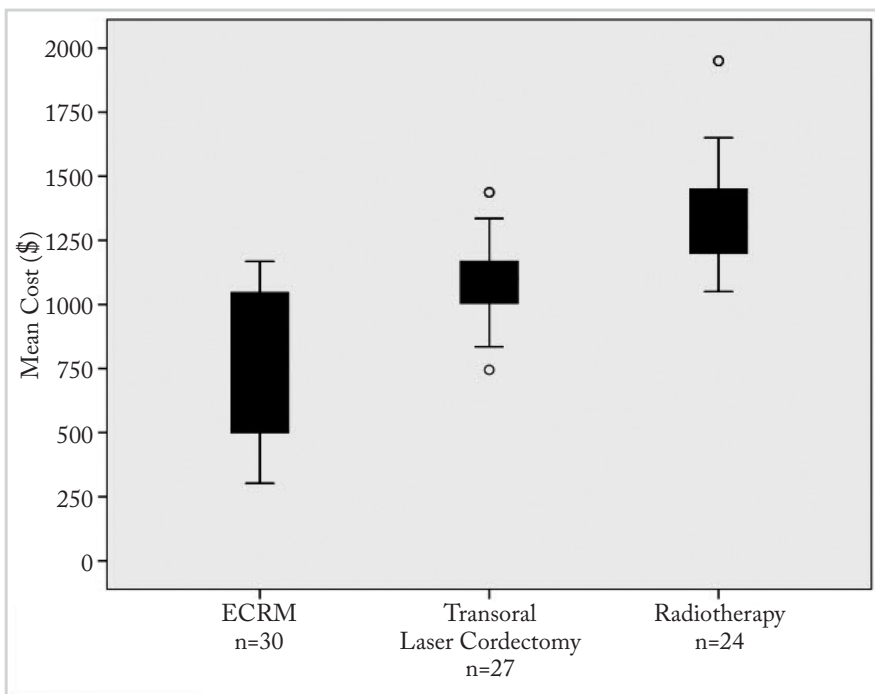

Figure 1. Mean cost of treatment modalities for early glottis cancer. ECRM has a significantly lower cost than other treatment procedures $(\mathrm{p}<0.05)$

ECRM: Endoscopic Cordectomy Using Radiofrequency Microelectrodes, USD: \$

\section{Cost-Effectiveness}

The mean cost of the procedures were as follows; $\$ 767.85 \pm 308.83$ for ECRM, $\$ 1.086 .95 \pm 250.22$ for transoral laser cordectomy, and $\$ 1.350 \pm 250.22$ for radiotherapy (Figure 1 ). The mean cost of ECRM was significantly lower than radiotherapy and transoral laser cordectomy ( $\mathrm{p}<0.05$ for both). The length of hospital stay after ECRM was $1.46 \pm 0.5$ days. The length of hospital stay after transoral laser cordectomy was $2.4 \pm 0.57$ days. The length of hospital stay after ECRM was found statistically significantly shorter than after transoral laser cordectomy $(\mathrm{p}<0.05)$. The mean duration of treatment for radiotherapy was $32.1 \pm 1.7$ days.

\section{Discussion}

The selection criteria of treatment modalities for early glottic cancers include post-treatment voice quality, cure rate, larynx preservation rate, morbidity, and treatment cost (1). In our study, which included 81 patients with T1a glottic squamous cell carcinomas, we demonstrated that the functional outcome of ECRM is similar to that of transoral laser cordectomy, but not as comparable as primary radiotherapy.

We have shown in our study that the perceptual voice quality of subjects that underwent radiotherapy was better than those that underwent endoscopic or transoral laser cordectomy. A possi- ble reason for this is that ECRM surgery and transoral laser cordectomy in T1a tumors correspond to type 3 cordectomy (transmuscular cordectomy) according to the ELS classification. Poorer voice quality after greater resections has been previously reported by some authors $(9,10)$. In a recent, and first, randomized study, Aaltonen et al. (11) have shown that the overall voice quality achieved by transoral laser cordectomy and radiotherapy was roughly similar after treatments, however patients treated with radiotherapy had less breathy voice.

When comparing parameters of aerodynamic voice analysis between the three groups in our study, we have shown that maximum phonation time was significantly longer in subjects undergoing radiotherapy. In their meta-analysis, Abdurehim et al. (2) have compared laser cordectomy and radiotherapy in terms of maximum phonation time, and found no statistically significant difference, but an obvious trend toward in favor of radiotherapy.

In our study, there was no significant difference between patients treated with ECRM or transoral laser cordectomy and those treated with radiotherapy with respect to mean fundamental frequencies $\left(\mathrm{F}_{0}\right.$ in Hertz $\left.[\mathrm{Hz}]\right)$, percentage of jitter, and percentage of shimmer. Results of a previous meta-analysis in the subgroup of $\mathrm{F}_{0}$ were reported to be in favor of radiotherapy (2). With respect to jitter and shimmer, the overall pooled results in the same meta-analysis showed no significant difference with laser cordectomy, but there was a slight trend in favor of radiotherapy (2).

In the literature, many studies are reported to have compared sound quality after endoscopic laser surgery or radiotherapy. In their meta-analysis, Abdurehim et al. (2) report no significant differences between the acoustic analysis parameters of patients treated with both endoscopic laser surgery and radiotherapy, but larynx preservation rate was significantly higher in patients initially treated with endoscopic laser cordectomy. Another recent systematic review (12) has indicated that greater resections with laser clearly showed poorer voice quality than those in lesser resections.

Endoscopic cordectomy using radiofrequency microdissection has been defined by Basterra et al. (5) in 2006 for the treatment of early glottic cancers, as an alternative to $\mathrm{CO}_{2}$ laser. The ECRM technique has many advantages compared to endoscopic laser cordectomy. The microelectrodes used in the ECRM technique can be reused, and no special equipment other than the 
microelectrodes are required. Laser surgery requires an experienced surgical team, safety precautions and special instruments whereas radiofrequency arrowtip electrodes do not require any of these means. The radiofrequency tips are reusable. We have been performing ECRM at our clinic since 2012 for the treatment of T1a glottic tumors. In our experience, ECRM is a useful and inexpensive alternative to laser cordectomy. As stated by Basterra et al. (13), the microelectrode device is very simple to handle, and the system is familiar to surgical teams. The cost of the equipment and the microelectrode is overwhelmingly low. Radiotherapy, on the other hand, has always been another treatment choice for our patients. Good local control rates and functional outcomes have been achieved with this therapy; however, the tumor-free laryngeal structures are also exposed to radiation have always been a matter of concern (14). Narayana et al. (15) performed a study in patients with successfully treated glottic cancer (stage 1a) and found that the frequency of a second primary tumor in the $5^{\text {th }}$, $10^{\text {th }}$ and $15^{\text {th }}$ years were $23 \%, 44 \%$ and $48.7 \%$, respectively. New techniques such as single cord irradiation have been offered for sparing critical perilaryngeal tumor-free structures $(14,16)$. Another major disadvantage of radiotherapy is that the duration of the treatment is longer. Type 3 cordectomy using radiofrequency microelectrodes resulted in a poorer voice quality than radiotherapy; however, it should be kept in mind that ECRM is a highly focused treatment for a clinical target volume, while conventional radiotherapy affects both the vocal cords and the other surrounding structures. The cost and duration of radiotherapy is also significantly higher than ECRM.

Our study has some limitations. As stated in the methods section, the choice between the three treatment modalities was not randomized but depended on the preference of the surgeon or the patient, and the availability of radiofrequency electrodes. We should also note that since we were not able to evaluate the patients' subjective and objective measurements preoperatively, a comparison between the groups in terms of the amount of post-treatment change in these parameters was not possible.

\section{Conclusion}

The results of our study revealed that maximum phonation time after radiotherapy treatment is longer than those of ECRM and transoral laser cordectomy. Percent jitter, percent shimmer, and fundamental frequency $\left(\mathrm{F}_{0}\right)$ after radiotherapy were significantly different than those of ECRM and transoral laser cordectomy. Furthermore, the ECRM group had lower costs and shorter hospital stay times than the other treatment modalities. According to these findings, ECRM seems to be advantageous because of the lower cost of treatment and hospitalization period in patients with early stage laryngeal cancer. Nevertheless, radiotherapy appears to be a more suitable treatment option in patients where sound quality is more important.

Ethics Committee Approval: Ethics committee approval was received for this study from the Ethics Committee of University of Health Sciences Ümraniye Training and Research Hospital (Date: 11/05/2015, No: 7389)

Informed Consent: Written informed consent was obtained from pa- tients who participated in this study.

Peer-review: Externally peer-reviewed.

Author Contributions: Concept - C.O., M.S.; Design - M.S., C.O.; Supervision - C.O., Ö.Y.; Resource - M.S., İ.D.; Materials - A.V.S., B.C.B., İ.D.; Data Collection and/or Processing - M.S., İ.D., B.K.; Analysis and/or Interpretation - M.S.; Literature Search - İ.D., B.K.; Writing - M.S., A.A.Ş.Y.; Critical Reviews - C.O., A.A.Ş.Y., Ö.Y.

Conflict of Interest: The authors have no conflicts of interest to declare.

Financial Disclosure: The authors declared that this study has received no financial support.

\section{References}

1. Higgins KM. What treatment for early-stage glottic carcinoma among adult patients: $\mathrm{CO} 2$ endolaryngeal laser excision versus standard fractionated external beam radiation is superior in terms of cost utility? Laryngoscope 2011; 121: 116-34. [CrossRef]

2. Abdurehim Y, Hua Z, Yasin Y, Xukurhan A, Imam I, Yuqin F. Transoral laser surgery versus radiotherapy: systematic review and meta-analysis for treatment options of T1a glottic cancer. Head Neck 2012; 34: 23-33. [CrossRef]

3. Yoo J, Lacchetti C, Hammond JA, Gilbert RW, Head and Neck Cancer Disease Site Group. Role of endolaryngeal surgery (with or without laser) versus radiotherapy in the management of early (T1) glottic cancer: a systematic review. Head Neck 2014; 36: 1807-19. [CrossRef]

4. Greulich MT, Parker NP, Lee P, Merati AL, Misono S. Voice outcomes following radiation versus laser microsurgery for T1 glottic carcinoma: systematic review and meta-analysis. Otolaryngol Head Neck Surg 2015; 152: 811-9. [CrossRef]

5. Basterra J, Frías S, Alba JR, Zapater E. A new device for treating laryngeal carcinoma using microdissection electrodes. Laryngoscope 2006; 116: 2232-4. [CrossRef]

6. Zapater E, Frías S, Pérez A, Basterra J. Comparative study on chronic tissue damage after cordectomies using either $\mathrm{CO} 2$ laser or microdissection electrodes. Head Neck 2009; 31: 1477-81. [CrossRef]

7. Basterra J, Zapater E, Moreno R, Hernández R. Electrosurgical endoscopic cordectomy with microdissection electrodes: a comparative study with CO2 laser. J Laryngol Otol 2006; 120: 661-4. [CrossRef]

8. Remacle M, Eckel HE, Antonelli A, Brasnu D, Chevalier D, Friedrich G, et al. Endoscopic cordectomy. A proposal for a classification by the Working Committee, European Laryngological Society. Eur Arch Otorhinolaryngol 2000; 257: 227-31. [CrossRef]

9. Roh JL, Kim DH, Kim SY, Park CI. Quality of life and voice in patients after laser cordectomy for Tis and T1 glottic carcinomas. Head Neck 2007; 29: 1010-6 [CrossRef]

10. Rydell R, Schalén L, Fex S, Elner A. Voice evaluation before and after laser excision vs. radiotherapy of T1A glottic carcinoma. Acta Otolaryngol 1995; 115: 560-5. [CrossRef]

11. Aaltonen LM, Rautiainen N, Sellman J, Saarilahti K, Mäkitie A, Rihkanen $\mathrm{H}$, et al. Voice quality after treatment of early vocal cord cancer: a randomized trial comparing laser surgery with radiation therapy. Int J Radiat Oncol Biol Phys 2014; 90: 255-60. [CrossRef]

12. van Loon Y, Sjögren EV, Langeveld TP, Baatenburg de Jong RJ, Schoones JW, van Rossum MA. Functional outcomes after radiotherapy or laser surgery in early glottic carcinoma: a systematic review. Head Neck 2012; 34: 1179-89. [CrossRef] 
Sürmeli et al.

13. Basterra J, Reboll R, Zapater E. Eighty-three cases of glottic and supraglottic carcinomas (stage T1-T2-T3) treated with transoral microelectrode surgery: how we do it. Clin Otolaryngol 2011; 36: 500-4. [CrossRef]

14. Osman SO, Astreinidou E, de Boer HC, Keskin-Cambay F, Breedveld S, Voet P, et al. IMRT for image-guided single vocal cord irradiation. Int J Radiat Oncol Biol Phys 2012; 82: 989-97. [CrossRef]
15. Narayana A, Vaughan AT, Fisher SG, Reddy SP. Second primary tumors in laryngeal cancer: results of long-term follow-up. Int J Radiat Oncol Biol Phys 1998; 42: 557-62. [CrossRef]

16. Levendag PC, Teguh DN, Keskin-Cambay F, Al-Mamgani A, van Rooij P, Astreinidou E, et al. Single vocal cord irradiation: a competitive treatment strategy in early glottic cancer. Radiother Oncol 2011; 101: 415-9. [CrossRef] 